\title{
Large photo-induced index variations in chalcogenide-on-silicon waveguides
}

\author{
R. Califa, ${ }^{1}$ Y. Kaganovskii, ${ }^{2}$ D. Munk, ${ }^{1}$ H. Genish, ${ }^{2}$ I. Bakish, ${ }^{1}$ M. Rosenbluh, ${ }^{2}$ and A. Zadok ${ }^{1, *}$ \\ ${ }^{1}$ Faculty of Engineering, Bar-Ilan University, Ramat-Gan 5290002, Israel \\ ${ }^{2}$ Department of Physics, Faculty of Exact Sciences, Bar-Ilan University, Ramat-Gan 5290002, Israel \\ ${ }^{*}$ Corresponding author: Avinoam.Zadok@biu.ac.il
}

Received June 16, 2014; revised July 27, 2014; accepted September 5, 2014;

posted September 11, 2014 (Doc. ID 214199); published October 10, 2014

\begin{abstract}
The postfabrication modification of the group delay in silicon-photonic waveguides is proposed, simulated and demonstrated experimentally. Group delay variations of $2 \%$ are achieved through photo-induced changes to an upper cladding layer of photosensitive $\mathrm{As}_{10} \mathrm{Se}_{90}$ chalcogenide glass. The illumination of the cladding layer by intense green light for a few seconds leads to mass transfer and removal of material, away from irradiated regions. The phenomenon is employed in the localized removal of the cladding layer from above the core region of a silicon-oninsulator waveguide, thereby modifying its phase and group delays. Using the proposed method, the free spectral range of a chalcogenide-on-silicon Mach-Zehnder interferometer was modified by $1 \%$. The technique is applicable to the postfabrication adjustment of the frequency response of silicon-photonic filters, comprised of several cascaded elements. (c) 2014 Optical Society of America

OCIS codes: (130.2755) Glass waveguides; (130.3130) Integrated optics materials; (230.7390) Waveguides, planar. http://dx.doi.org/10.1364/OL.39.005905
\end{abstract}

Communication among large-scale computer systems relies increasingly on photonic means to accommodate ever-expanding bandwidth demands [1]]. Implementation of photonics on the silicon-on-insulator (SOI) material platform holds promise for large-scale, low-cost integration between photonics and microelectronics [2], in optical communication and signal processing applications [3-5]. One of the primary tasks carried out by photonic integrated circuits (PICs) is the multiplexing and de-multiplexing of high-bandwidth data channels. Multiplexing can be performed by silicon-photonic filters, comprised of a cascade of Mach-Zehnder interferometers (MZIs) and ring resonators [3]. The proper function of such filters depends on precise phase and group delays of individual optical paths, which are difficult to achieve in open-loop fabrication [6]. Hence, procedures for the postfabrication trimming of optical paths are mandatory.

Optical trimming procedures can be broadly classified in two categories: active dynamic tuning, and permanent post fabrication trimming. Common active solutions rely on pre-patterned metallic heaters $[\underline{7}, \underline{8}]$, or on injection of free carriers into p-i-n junction structures [9]. Active tuning approaches add to the complexity of device fabrication, require continuous closed-loop feedback, and may also require continuous power consumption. One-time, permanent postfabrication trimming is preferable when the device temperature is sufficiently stabilized.

Numerous mechanisms have been applied toward permanent postfabrication trimming of PICs [10-17]. Examples include irradiation with intense ultraviolet or visible light $[\underline{10}, \underline{11}]$, use of liquid crystals [12], photoinduced stresses, and photo-elasticity [13]. Other methods include patterning of a thin, upper silicon nitride film [16] and local oxidation of the surface of silicon [17]. Most previous reports deal with phase delay modifications, whereas trimming of the group delay is not often addressed. Group delay modifications are required, however, for example in the adjustment of free spectral ranges (FSRs) of MZI filters.
Chalcogenide glasses are amorphous semiconducting materials, characterized by a broad transparency window, high refractive index, and pronounced optical nonlinearities [18-20]. Chalcogenide glasses also exhibit numerous permanent photo-induced modification effects [21,22], ranging from refractive index variations [23] to material reflow and mass transfer [24]. Photosensitivity is used in direct lithography and in trimming of MZIs and ring resonators fabricated in chalcogenide glasses $[25,26]$. Chalcogenide glasses also serve as a photosensitive upper cladding of waveguides [27]. Recently, Canciamilla and co-workers modified the transfer functions of chalcogenide-on-SOI ring resonators, through relatively low-power illumination of the upper cladding with near-bandgap light [15]. The resultant photodarkening of the upper cladding modified the effective index of the resonator waveguide by 0.016 refractive index units (RIU) [15].

In this work, we employ photo-induced mass transfer of an $\mathrm{As}_{10} \mathrm{Se}_{90}$ upper cladding layer in the broad post fabrication tuning of SOI waveguides. Mass transfer is induced by localized illumination with green laser light of $1 \mathrm{MW} / \mathrm{cm}^{2}$ intensity. Changes to the group delay index by $0.07 \mathrm{RIU}$ are achieved through selective photo-removal of the upper cladding, from above the core of an underlying SOI ridge waveguide, along sections of one arm of a MZI. The FSR of the MZI is modified by $1 \%$, while the duration of the trimming process is only a few seconds. Preliminary results were briefly presented in [28].

The cross-section of waveguides used in this work is shown in Fig. 1(top). Cores of ridge waveguides are patterned in the $2 \overline{2} 0$-nm-thick silicon device layer of an SOI wafer, which is partially etched to a residual thickness of $150 \mathrm{~nm}$. The width of the waveguide core is $700 \mathrm{~nm}$. The device layer is covered by a 60 -nm-thick silica buffer layer, followed by a 700-nm-thick $\mathrm{As}_{10} \mathrm{Se}_{90}$ layer. The refractive index of $\mathrm{As}_{10} \mathrm{Se}_{90}$ at $1550 \mathrm{~nm}$ is $2.35 \mathrm{RIU}$. The ridge cross-section was chosen for convenience, but other geometries, having stronger waveguide dispersion, would give rise to even larger index variations. 


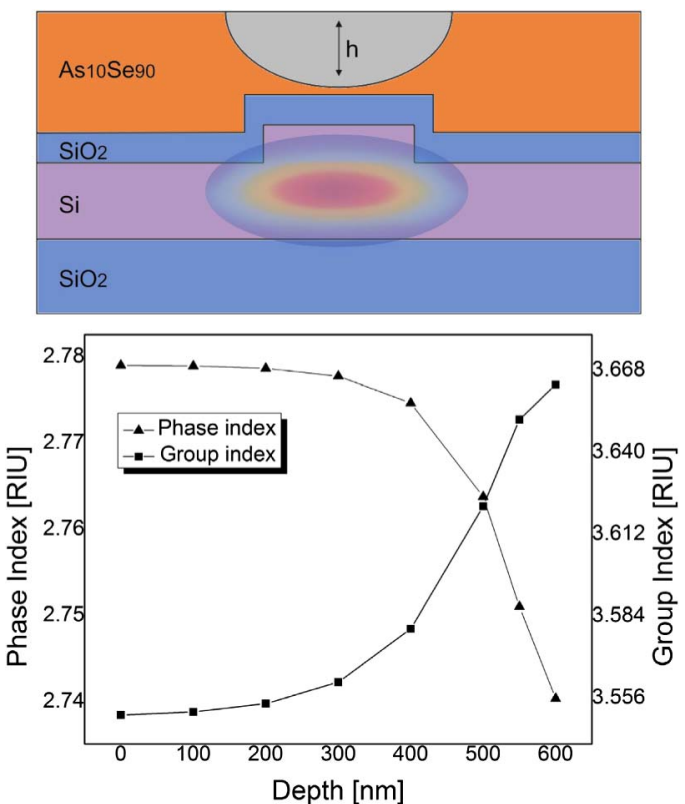

Fig. 1. Top: schematic drawing of the cross-section of a SOI ridge waveguide, with a silica buffer layer and upper layer of chalcogenide glass, and of the profile of the fundamental transverse electric (TE) mode (not to scale). The cross-section illustrates the localized photo-removal of the upper cladding layer, reducing its thickness by a depth $h$. Bottom: calculated effective index (triangle symbols) and group velocity index (square symbols) of the TE mode, as a function of $h$.

Photo-induced mass transfer is used to partially remove the chalcogenide layer from above the silicon core, reducing the thickness of the upper cladding by $h \mathrm{~nm}$ [Fig. 1 (top)]. The effective index of the transverse electric ( $\overline{\mathrm{TE}}$ ) mode $n_{\mathrm{ph}}$ was calculated over a range of wavelengths $\lambda$ around $1550 \mathrm{~nm}$ using COMSOL multi-physics. Figure 1 (bottom) shows the calculated $n_{\mathrm{ph}}$ at $1550 \mathrm{~nm}$ as a function of $h$. Complete removal of the upper cladding layer is expected to modify $n_{\text {ph }}$ by 0.04 RIU. The expected modifications of $n_{\mathrm{ph}}$ are relatively modest, as the removal of the upper cladding is counteracted by stronger modal confinement in the silicon ridge. The figure also shows the calculated group index, defined as: $n_{g} \equiv n_{\mathrm{ph}}-$ $\lambda \cdot d n_{\mathrm{ph}} / d \lambda$. The group delay along waveguides is governed by $n_{g}$. The calculated changes in $n_{g}$ are 0.11 RIU for complete removal. Simulations suggest that much larger changes in $n_{g}$ could be obtained using the fundamental transverse magnetic (TM) mode; however, the coupling of light into devices used in this work does not support the TM mode.

For comparison, the expected changes in $n_{\mathrm{ph}}$ and $n_{g}$ following photo-darkening of the upper cladding layer were calculated as well $[15,23]$. An extreme increase in the upper cladding index by 0.3 RIU [29], for example, would modify the $n_{\mathrm{ph}}$ and $n_{g}$ by $0.026 \mathrm{RIU}$ and $0.089 \mathrm{RIU}$, respectively. Both values are smaller than the corresponding modifications associated with photo-induced structural changes.

Postfabrication trimming of the group delay was experimentally demonstrated using SOI MZIs with a differential path length of $1 \mathrm{~mm}$. The thicknesses of the silicon handle layer, $\mathrm{SiO}_{2}$ layer, and silicon device layer were $750 \mu \mathrm{m}, 2 \mu \mathrm{m}$, and $220 \mathrm{~nm}$, respectively. Cores were defined through electron-beam lithography (CRESTEC CABEL 9000C instrument and ZEP 520a resist), and subsequent reactive ion etching to a depth of $70 \mathrm{~nm}$, the value used in simulations. Etching was performed using a mixture of $\mathrm{SF}_{6}(65 \mathrm{sccm})$ and $\mathrm{C}_{4} \mathrm{~F}_{8}(10 \mathrm{sccm})$ gasses at $37.5 \mu \mathrm{Pa}$ pressure, with a plasma radio-frequency power of $100 \mathrm{~W}$ for 18 seconds. Vertical grating couplers were patterned at the termini of the waveguides leading to the MZIs for the coupling of light to/from standard singlemode fibers.

A 60-nm-thick silica buffer layer was deposited above the MZIs (while protecting the gratings areas), using photo-lithography, electron-beam evaporation, and liftoff of the photo-resist. Evaporation was carried out at $9 \mu \mathrm{Pa}$ and at a rate of $0.03 \mathrm{~nm} / \mathrm{s}$. The buffer layer was necessary to reduce additional losses following the deposition of the chalcogenide glass layer. The cause of these excess losses is still under investigation. Lastly, a 700-nm-thick upper cladding of $\mathrm{As}_{10} \mathrm{Se}_{90}$ was applied on top of the buffer layer, using a second photo-lithography stage and thermal evaporation at a vacuum of $0.3 \mu \mathrm{Pa}$ and at a rate of $0.5 \mathrm{~nm} / \mathrm{s}$. The specific composition was chosen due to its known strong photo-induced mass transfer, low glass transition temperature $\left(T_{g}\right)$ of $100^{\circ} \mathrm{C}$, relative stability when exposed to air, and weak photodarkening at room temperature [24,30]. Other compositions, such as ternary compounds of Ga-As-Se or $\mathrm{Ga}-\mathrm{Sb}-\mathrm{Se}$, could be used instead; however, these compositions were not available to us. Figure 2 shows a topview optical microscope image of a sample containing several MZIs. Regions deposited with silica and $\mathrm{As}_{10} \mathrm{Se}_{90}$ can be identified.

Transmission losses of MZIs were measured following initial fabrication in SOI, after the deposition of silica, and following the deposition of $\mathrm{As}_{10} \mathrm{Se}_{90}$. The end-toend loss of as-fabricated SOI MZIs was $19 \mathrm{~dB}$. The grating coupling losses were $16 \mathrm{~dB}$, estimated by auxiliary measurements of end-to-end losses of waveguides having different lengths. Losses increased by $5 \mathrm{~dB}$ following the deposition of the silica and chalcogenide layers. Excess losses are a drawback of the proposed method, and means to eliminate them are currently under study.

Spectral transfer functions were characterized using an optical vector network analyzer (LUNA OVAe8000), with 2.5-pm resolution. Table 1 lists the measured FSRs following the three fabrication stages. The expected FSRs based on numerical simulations are listed

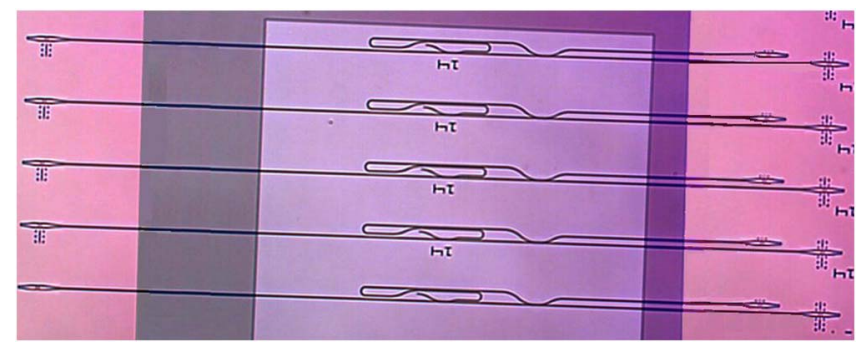

Fig. 2. Top-view optical microscope image of a sample containing chalcogenide-on-SOI MZIs, terminated by vertical grating couplers. The outer, darker square region is covered by an intermediate silica buffer layer. The inner square region is also deposited with an upper cladding layer of chalcogenide glass. 
Table 1. Simulated and Measured Group Indices and FSRs of MZIs Following Different Fabrication Steps

\begin{tabular}{|c|c|c|c|}
\hline & $\begin{array}{c}\text { Simulated } \\
\text { Group } \\
\text { Index }\end{array}$ & $\begin{array}{c}\text { Expected } \\
\text { MZI FSR } \\
\quad(\mathrm{nm})\end{array}$ & $\begin{array}{c}\text { Measured } \\
\text { MZI FSR } \\
\quad(\mathrm{nm})\end{array}$ \\
\hline SOI only & 3.6649 & 0.655 & 0.642 \\
\hline Following silica deposition & 3.6571 & 0.657 & 0.644 \\
\hline $\begin{array}{l}\text { Following } \mathrm{As}_{10} \mathrm{Se}_{90} \\
\text { deposition }\end{array}$ & 3.5444 & 0.678 & 0.668 \\
\hline Following mass transfer & & 0.667 & 0.661 \\
\hline
\end{tabular}

as well. The deposition of the upper layers increased the FSR by $26 \mathrm{pm}$, in good agreement with simulations. The small, constant offset between measured and calculated FSRs is due to uncertainty in the refractive index of the $\mathrm{As}_{10} \mathrm{Se}_{90}$ layer, and possible variations in the silicon core width.

Photo-induced mass transfer of the upper chalcogenide layer was carried out using a laser at 532-nm wavelength, coupled through an objective lens of $\times 50$ magnification. The $20-\mathrm{mW}$ laser power was focused to a spot-size diameter of $1.5 \mu \mathrm{m}$ (intensity of $\sim 1 \mathrm{MW} / \mathrm{cm}^{2}$ ), above the core of the SOI waveguide of the long MZI arm. The sample was scanned under the beam by a motorized linear stage at $200 \mu \mathrm{m} / \mathrm{s}$ speed. The device was aligned so that a $560-\mu \mathrm{m}$-long segment of the long arm of the MZI was irradiated. The laser energy associated with the process was $60 \mathrm{~mJ}$. The minimum feature size is set by the diffraction limit, whereas the maximum size is only limited by scanning mechanics and process duration. Figure 3 (top) shows a top-view

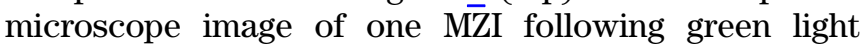
illumination.

Figure 3 (center and bottom) shows a two-dimensional height scan and a cross-section profile of part of the illuminated region, taken by an atomic force microscope. The 700-nm-thick $\mathrm{As}_{10} \mathrm{Se}_{90}$ layer is removed from a $3-\mu \mathrm{m}$ wide region almost entirely [24]. Note that the removed glass material is transferred and partially piled to the sides of the illuminated region [24]. Mass transfer is due to viscous flow in the glass: absorption raises the temperature of illuminated regions above the transition temperature $T_{g}$ of the material. The temperature gradient initiates a fast lateral flow of the liquid layer, from hot to cold regions, in the direction normal to the forming groove [31,32]. Figure 4 shows a focused ion beam (FIB) cross-section of the illuminated area. The residual thickness of the $\mathrm{As}_{10} \mathrm{Se}_{90}$ layer above the waveguide core was reduced to about $50 \mathrm{~nm}$ across a $3-\mu \mathrm{m}$-wide region.

Figure 5 shows the measured power transfer functions of the MZI before and after photo-induced mass transfer. The FSR of the device is reduced by $6.8 \mathrm{pm}$, or $1 \%$ (see Table 1). This change corresponds to a modification of the average group index in the 560 - $\mu$ m-long illuminated segment of the MZI long arm by 0.07 RIU, or $2 \%$. Simulations suggest a larger variation in FSR, by as much as $10.5 \mathrm{pm}$ (see Table 1). The most likely source of this discrepancy is an angular misalignment between the waveguide core layout and the scanning trajectory of the device through the green light spot. Angular misalignment of $0.5^{\circ}$, for example, would reduce the length of the

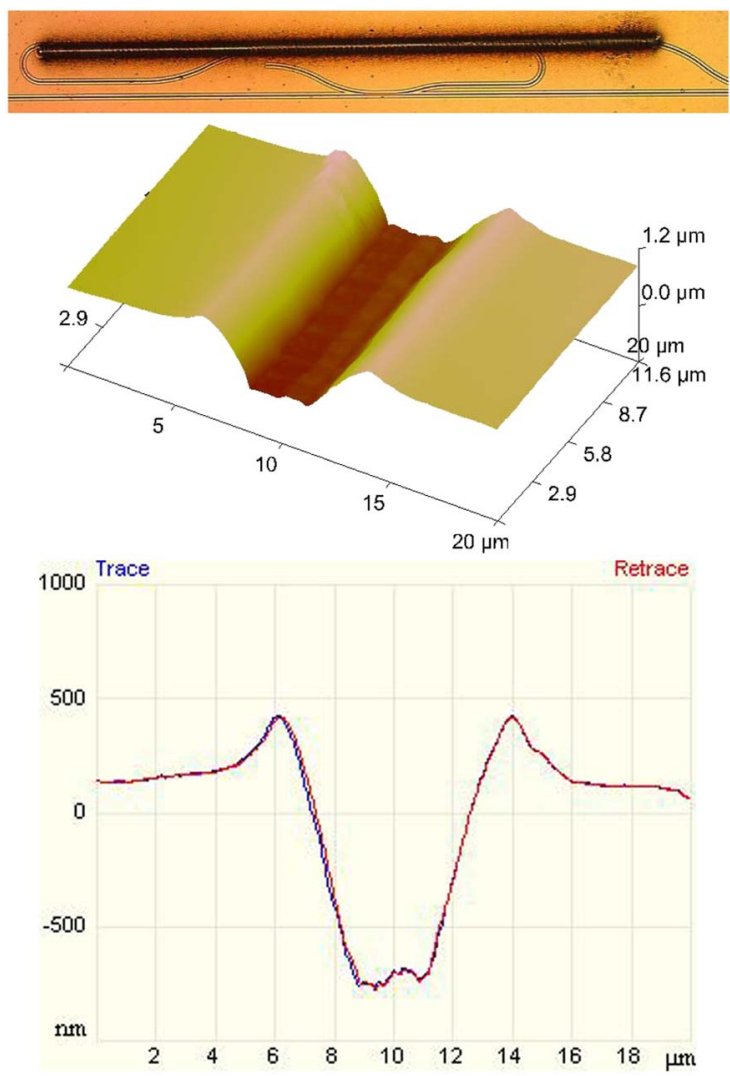

Fig. 3. Top: top-view microscope image of a chalcogenide-onSOI MZI, following the illumination of part of the long arm by green laser light. Photo-removal of the upper chalcogenide layer is evident in the illuminated region. Center: atomic force microscope scan of part of the illuminated region, showing near-complete removal of the chalcogenide layer. Bottom: height cross-sections taken from the map of the central panel.

waveguide within the MZI arm where the group index is effectively modified from 560 to $350 \mu \mathrm{m}$, and reduce the change in FSR to $6.8 \mathrm{pm}$. Changes to the end-to-end loss of the device following trimming were below the measurement uncertainty of $\pm 1 \mathrm{~dB}$. The extinction ratios before and after exposure were 14 and $16 \mathrm{~dB}$, respectively.

In conclusion, the rapid postfabrication trimming of the group delay in a chalcogenide-on-silicon MZI was demonstrated. A change in FSR by $1 \%$ was achieved through photo-induced mass transfer in an $\mathrm{As}_{10} \mathrm{Se}_{90}$ cladding layer. The method is applicable to complex filter designs, consisting of multiple, cascaded MZIs and/or

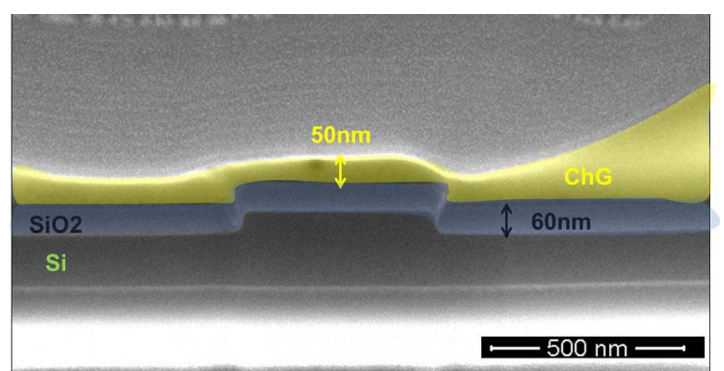

Fig. 4. FIB cross section of a chalcogenide-on-SOI waveguide, following the photo-induced mass transfer of the upper chalcogenide cladding layer from above the silicon core. The silica and chalcogenide layers are indicated in false colors. 


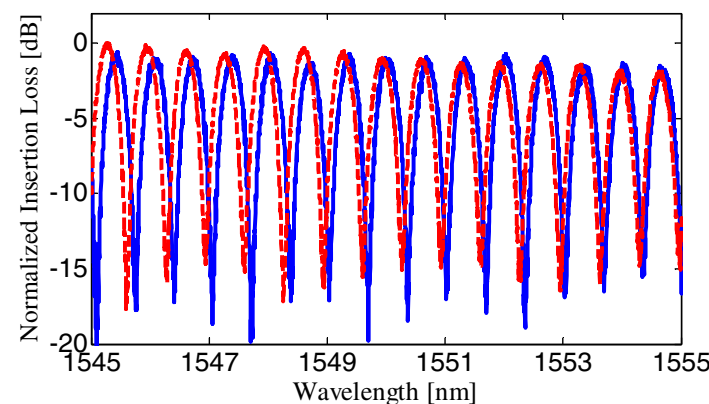

Fig. 5. Measured power transfer of a chalcogenide-on-SOI MZI, before (red, dashed) and after (blue, solid) the photoremoval of the upper $\mathrm{As}_{10} \mathrm{Se}_{90}$ layer above the waveguide core in part of the long arm. The FSR of the MZI is reduced by $6.8 \mathrm{pm}$ $(1 \%)$.

resonators. The choice of low- $T_{g}$ cladding material helps reduce the illumination intensity necessary for mass transfer; however, the long-term stability of the device might be compromised. Changes in the FSR of trimmed devices over a period of one month were below the measurement uncertainty of $\pm 0.1 \%$. Long-term stability of is still under study. Stability can be improved using compositions with higher $T_{g}$, and through the additional deposition of thin, protective capping layers such as $\mathrm{SiO}_{2}$ or $\mathrm{Al}_{2} \mathrm{O}_{3}$. Future work will also address larger photoinduced index changes in fully-etched silicon nanowires and using TM modes, and the implementation of a closedloop trimming and characterization setup.

R. Califa, D. Munk, and A. Zadok acknowledge the support of the Israeli Science Foundation (ISF) through Grant No. 635/10, and the TERA SANTA consortium of the Chief Scientist Office, the Israeli Ministry of Economics. The authors acknowledge the support of the Bar-llan Institute of Nano-Technology and Advanced Materials (BINA), Dr. Y. Abulafia, Dr. M. Feldberg, and Dr. Y. Fleger in the fabrication and analysis of devices.

\section{References}

1. G. T. Reed and A. P. Knights, Silicon Photonics: An Introduction (Wiley, 2004).

2. R. Soref, IEEE J. Sel. Top. Quantum Electron. 12, 1678 (2006).

3. C. K. Madsen and J. H. Zhao, Optical Filter Design and Analysis: A Signal Processing Approach (Wiley, 1999).

4. M. R. Watts, W. A. Zortman, D. C. Trotter, R. W. Young, and A. L. Lentine, Opt. Express 19, 21989 (2011).

5. D. Hillerkuss, R. Schmogrow, T. Schellinger, M. Jordan, M. Winter, G. Huber, T. Vallaitis, R. Bonk, O. Kleinow, F. Frey, M. Roeger, S. Koeing, A. Ludwig, A. Marculescu, J. Li, M. Hoh, M. Dreschmann, J. Meyer, S. Ben Ezra, N. Narkiss, B. Nebendahl, F. Paramigiani, P. Petropoulos, B. Resan, A. Oehler, K. Weingarten, T. Ellenmeyer, J. Lutz, M. Moeller, M. Huebner, J. Becker, C. Koos, W. Freude, and J. Leuthold, Nat. Photonics 5, 364 (2011).

6. W. A. Zortman, D. C. Trotter, and M. R. Watts, Opt. Express 18, 23598 (2010).
7. P. Dong, W. Qian, H. Liang, R. Shafiiha, D. Feng, G. Li, J. E. Cunningham, A. V. Krishnamoorthy, and M. Asghari, Opt. Express 18, 20298 (2010).

8. F. Gan, T. Barwicz, M. A. Popovic, M. S. Dahlem, C. W. Holzwarth, P. T. Rakich, H. I. Smith, E. P. Ippen, and F. X. Kartner, in Photonics in Switching (IEEE, 2007), p. 67.

9. H. Lira, S. Manipatruni, and M. Lipson, Opt. Express 17, 22271 (2009).

10. H. Haeiwa, T. Naganawa, and Y. Kokubun, IEEE Photon. Technol. Lett. 16, 135 (2004).

11. L. Zhou, K. Okamoto, and S. J. B. Yoo, IEEE Photon. Technol. Lett. 21, 1175 (2009).

12. S. Lambert, W. De Cort, J. Beeckman, K. Neyts, and R. Baets, Opt. Lett. 37, 1475 (2012).

13. J. Schrauwen, D. Van Thourhout, and R. Baets, Opt. Express 16, 3738 (2008).

14. G. D. Maxwell, R. Kashyap, and B. J. Ainslie, Electron. Lett. 28, 2106 (1992).

15. A. Canciamilla, F. Morichetti, S. Grillanda, P. Velha, M. Sorel, V. Singh, A. Agarwal, L. C. Kimerling, and A. Melloni, Opt. Express 20, 15807 (2012).

16. A. H. Atabaki, A. A. Eftekhar, M. Askari, and A. Adibi, Opt. Express 21, 14139 (2013).

17. Y. Shen, I. Divliansky, D. Basov, and S. Mookherjea, in Optical Fiber Communication Conference, OSA Technical Digest (CD) (Optical Society of America, 2011), paper PDPC3.

18. B. T. Kolomiets, Phys. Status Solidi B 7, 359 (1964).

19. R. Frerichs, J. Opt. Soc. Am. 43, 1153 (1953).

20. K. Ogusu, J. Yamasaki, S. Maeda, M. Kitao, and M. Minakata, Opt. Lett. 29, 265 (2004).

21. A. E. Owen, A. P. Firth, and P. J. S. Ewen, Philos. Mag. 52 (3), 347 (1985).

22. J. D. Musgraves, K. Richardson, and H. Jain, Opt. Mater. Express 1, 921 (2011)

23. A. Zoubir, M. Richardson, C. Rivero, A. Schulte, C. Lopez, and K. Richardson, Opt. Lett. 29, 748 (2004).

24. A. Saliminia, T. V. Galstian, and A. Villeneuve, Phys. Rev. Lett. 85, 4112 (2000).

25. X. Gai, T. Han, A. Prasad, S. Madden, D.-Y. Choi, R. Wang, D. Bulla, and B. Luther-Davies, Opt. Express 18, 26635 (2010).

26. N. Carlie, J. D. Musgraves, B. Zdyrko, I. Luzinov, J. Hu, V. Singh, A. Agarwal, L. C. Kimerling, A. Canciamilla, F. Morichetti, A. Melloni, and K. Richardson, Opt. Express 18, 26728 (2010).

27. W. C. Tan, Q. Chen, J. Kim, and C. Madsen, IEEE J. Quantum Electron. 48, 237 (2012).

28. R. Califa, Y. Kaganovskii, D. Munk, H. Genish, I. Bakish, M. Rosenbluh, and A. Zadok, in CLEO: 2014, OSA Technical Digest (online) (Optical Society of America, 2014), paper STh1M.7.

29. G. Pfeiffer, M. A. Paesler, and S. C. Agrawal, J. Non-Cryst. Solids 130, 111 (1991).

30. M. L. Trunov, P. M. Nagy, V. Takats, P. M. Lytvyn, S. Kokenyesi, and E. Kalman, J. Non-Cryst. Solids 355, 1993 (2009).

31. M. Lax, J. Appl. Phys. 48, 3919 (1977).

32. K. Shemesh, Y. Kaganovskii, and M. Rosenbluh, in Planar Waveguides and Other Confined Geometries: Theory, Technology, Production, and Novel Applications, G. Marowsky, ed. (Springer, 2014), Chap. 5. 